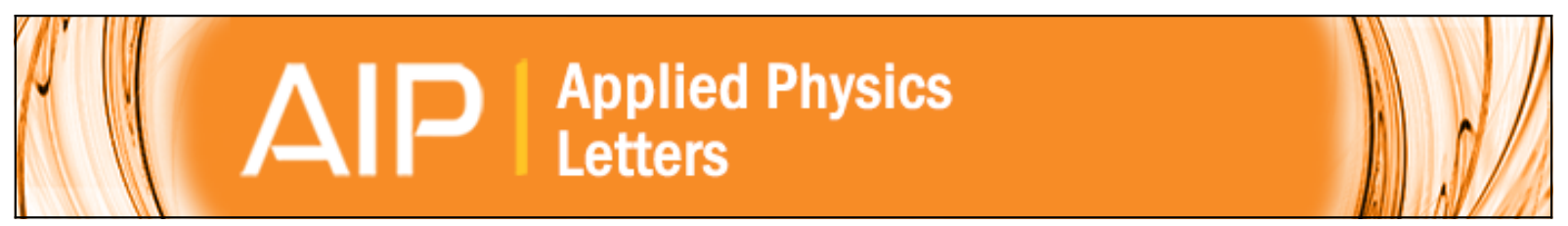

\title{
Transport properties of single TiO2 nanotubes
}

Markus Stiller, José Barzola-Quiquia, Israel Lorite, Pablo Esquinazi, Robin Kirchgeorg, Sergiu P. Albu, and

Patrik Schmuki

Citation: Applied Physics Letters 103, 173108 (2013); doi: 10.1063/1.4826640

View online: http://dx.doi.org/10.1063/1.4826640

View Table of Contents: http://scitation.aip.org/content/aip/journal/apl/103/17?ver=pdfcov

Published by the AIP Publishing

\section{Articles you may be interested in}

Improved visible solar absorber based on TiO2 nanotube film by surface-loading of plasmonic Au nanoparticles J. Appl. Phys. 114, 063510 (2013); 10.1063/1.4818441

Erratum: "Investigation of electrical transport in anodized single TiO2 nanotubes" [Appl. Phys. Lett. 102, 043105 (2013)]

Appl. Phys. Lett. 103, 039901 (2013); 10.1063/1.4815940

Electrical property measurements of $\mathrm{Cr}-\mathrm{N}$ codoped TiO2 epitaxial thin films grown by pulsed laser deposition Appl. Phys. Lett. 102, 172108 (2013); 10.1063/1.4804240

Investigation of electrical transport in anodized single $\mathrm{TiO} 2$ nanotubes

Appl. Phys. Lett. 102, 043105 (2013); 10.1063/1.4789763

Transport properties in single-crystalline rutile $\mathrm{TiO} 2$ nanorods

Appl. Phys. Lett. 99, 222107 (2011); 10.1063/1.3665635

\section{Asylum Research Atomic Force Microscopes}

Unmatched Performance, Versatility and Support

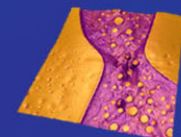

Coating Uniformity \& Roughness
Modulus of Polymers

\& Advanced Materials

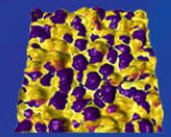

Nanoscale Conductivity

\& Permittivity Mapping
Piezoelectrics

\& Ferroelectrics

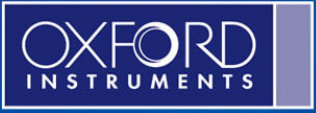

The Business of Science
+1 (805) 696-6466 sales@AsylumResearch.com www.AsylumResearch.com 


\title{
Transport properties of single $\mathrm{TiO}_{2}$ nanotubes
}

\author{
Markus Stiller, ${ }^{1}$ José Barzola-Quiquia, ${ }^{1}$ Israel Lorite, ${ }^{1}$ Pablo Esquinazi, ${ }^{1}$ Robin Kirchgeorg, ${ }^{2}$ \\ Sergiu P. Albu, ${ }^{2}$ and Patrik Schmuki ${ }^{2,3}$ \\ ${ }^{1}$ Division of Superconductivity and Magnetism, Institute for Experimental Physics II, University of Leipzig, \\ D-04103 Leipzig, Germany \\ ${ }^{2}$ Chair for Surface Science and Corrosion Department Material Science and Engineering, \\ University of Erlangen, D-91058 Erlangen, Germany \\ ${ }^{3}$ Department of Chemistry, King Abdulaziz University, Jeddah, Saudi Arabia
}

(Received 20 August 2013; accepted 9 October 2013; published online 22 October 2013)

\begin{abstract}
We investigated the electric transport properties of single $\mathrm{TiO}_{2}$ nanotubes separated from an anodic titania nanotube array. The temperature dependence of the resistance measured with the conventional four point method of all investigated samples show a Mott variable range hopping behavior. The results obtained with two contacts indicate the existence of a potential barrier between the $\mathrm{Cr} / \mathrm{Au}$ contacts and samples surfaces, which influence is clearly observable at temperatures $<150 \mathrm{~K}$. Impedance spectroscopy in the frequency range of $40 \mathrm{~Hz}$ to $1 \mathrm{MHz}$ carried out at room temperature indicates that the electronic transport of these polycrystalline tubes is dominated by the grain cores. (C) 2013 AIP Publishing LLC. [http://dx.doi.org/10.1063/1.4826640]
\end{abstract}

In the last decade, titanium dioxide nanotube arrays, formed by self-organizing anodization, have attracted considerable attention. Mainly these 1D structures are expected to replace titanium dioxide nanostructured particulate electrodes in catalysis, ${ }^{1}$ photocatalysis, ${ }^{2}$ dye-synthesized solar cells, ${ }^{3}$ gas sensors, ${ }^{4}$ or batteries, ${ }^{5}$ due to improved electron transfer properties. Electronic, chemical, and ionic properties of $\mathrm{TiO}_{2}$ depend considerably on the specific phase of $\mathrm{TiO}_{2} \cdot{ }^{6,7}$ Rutile and anatase are the most used phases in many applications of $\mathrm{TiO}_{2}$ based on charge transfer reactions (solar cells, photocatalysis). ${ }^{8-12}$ Nevertheless, the general mechanism for electron transport, particularly in nanostructures, is not well studied and understood. The transport properties can be strongly influenced by the scattering as well as through the possible increase of the carrier density at the grain boundaries, namely, in polycrystalline nanotube arrays. ${ }^{13}$ Additionally, the sample surfaces containing intergranular boundaries may play an extra role in the conductivity. In the present work, we investigated the conductivity of single $\mathrm{TiO}_{2}$ nanotubes separated from anodic $\mathrm{TiO}_{2}$ nanotube arrays. The relatively simple way to produce such self-organized $\mathrm{TiO}_{2}$ nanotubes on Ti substrates makes this approach cheap and efficient compared to other fabrication techniques. These $\mathrm{TiO}_{2}$ tubes are usually amorphous after growing but can be converted to anatase by annealing at temperatures $(T)$ higher than $300{ }^{\circ} \mathrm{C} .{ }^{14,15}$ After annealing, the nanotubes are polycrystalline with an average grain size of 10 to $50 \mathrm{~nm} .^{16,17}$ We have used impedance spectroscopy in order to discern between the mechanisms of conduction given by the possible electron pathways in the sample. ${ }^{18}$ To characterize the contact resistance we have also studied the current-voltage $(I-V)$ characteristics using two points measurements as recently reported for single $\mathrm{TiO}_{2}$ nanotubes. ${ }^{19}$

Prior to anodization the titanium foils (Advent Ltd., $99,6 \%, 0.1 \mathrm{~mm}$ ) were cleaned by sonificating them in acetone, ethanol, and deionized (DI) water and were then dried in a nitrogen stream. The $\mathrm{TiO}_{2}$ nanotubes were grown by anodization using a potentiostat (Jaissle IMP 88 PC) in a three electrode setup, where a platinum sheet acts as counter, a platinum wire as reference, and the Ti foil as working electrode. The anodization was carried out at room temperature in an ethylene glycol (Sigma Aldrich 99.5\%) $0.15 \mathrm{~mol} / \mathrm{l} \mathrm{am-}$ monium fluoride electrolyte (Sigma Aldrich) with $1 \mathrm{M} \mathrm{H}_{2} \mathrm{O}$ at $60 \mathrm{~V}$ for $10 \mathrm{~h}$. After anodization, the grown layers were immersed in ethanol for $1 \mathrm{~h}$ and dried in a nitrogen stream. To transfer the grown amorphous $\mathrm{TiO}_{2}$ nanotubes into a crystalline structure annealing at $350{ }^{\circ} \mathrm{C}$ and $450{ }^{\circ} \mathrm{C}$ was carried out in a tube furnace in air. After annealing, the tubes were removed from the Ti foil by sonfication in DI water.

The nanotubes were then transferred on the top of a $\mathrm{SiN}_{x} / \mathrm{SiO}_{2}$ substrate by a printing method, which means to press a $\mathrm{TiO}_{2}$ bundle between the two above mentioned substrates. After this step, the substrate is blown gently with air to remove big pieces and dirt, followed by an identification of suitable nanotubes. For this, we examined the nanotubes in a Dual Beam Microscope (FEI NanoLab XT 200) and selected a particular one, see example in Fig. 1(a). To fix the nanotube on the substrate, we used electron beam induced deposition (EBID), ${ }^{20}$ a technique based on decomposing gaseous molecules by electron beam, leading to deposition of non-volatile fragments of a given material, in our case $\mathrm{WC}_{x}$. Using the electron beam, instead of the ion beam induced deposition (IBID) usually with $\mathrm{Ga}^{+}$ions, we preserve the sample structure and intrinsic characteristics. The $\mathrm{WC}_{x}$ material deposited on four different places along the nanotube length, see Fig. 1(b), is nearly insulating ${ }^{21}$ and helps to fix the sample on the substrate and also facilitates the preparation of the electrical contacts at different positions along the sample, see Fig. 1(c). To do the electrical contacts, the substrate is covered with a resist (PMMA $950 \mathrm{~K}$, AR-P 671-05) for the electron beam lithography process. After developing the electrode shapes, $\mathrm{Cr} / \mathrm{Au}$ thin bilayer films of thickness of $5 \mathrm{~nm}$ and $35 \mathrm{~nm}$, respectively, were deposited by sputtering on the sample/PMMA/substrate arrangement. In the last step and after removing the rest of the PMMA, the sample is contacted to a chip carrier for the transport measurements. 

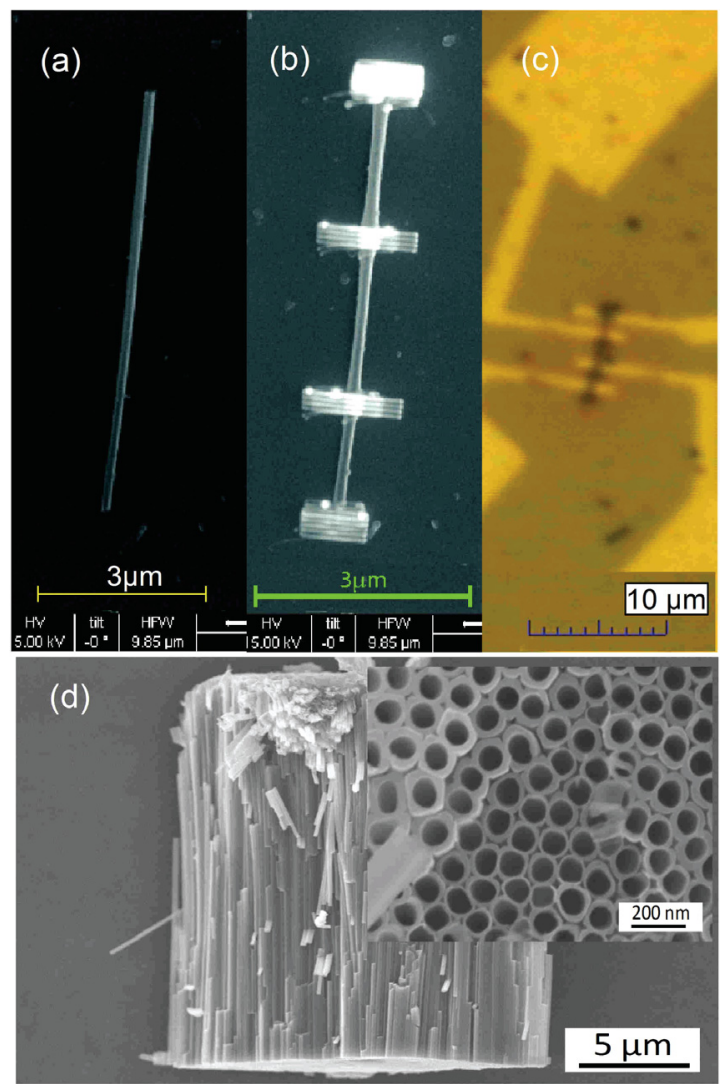

FIG. 1. (a) One $\mathrm{TiO}_{2}$ nanotube before fixing it on the substrate. (b) The nanotube fixed with $\mathrm{WC}_{x}$. (c) After producing the electrical contacts. (d) Scanning electron microscope side and top views (inset) of a bundle of $\mathrm{TiO}_{2}$ nanotubes.

The carrier is mounted on the cold head of a standard closed cycle refrigerator inside a vacuum bell, with a minimal $T \simeq 25 \mathrm{~K}$. The resistance measurements were performed using a current source (Keithley 6221) and a nanovoltmeter (Keithley 2182). Impedance measurements were carried out using an Agilent 4294A impedance analyzer, in the frequency range of $40 \mathrm{~Hz}$ to $1 \mathrm{MHz}$ at room temperature and in darkness to avoid changes due to the photoconductivity of the sample. We measured the resistance and reactance using alternating signals of $20 \mathrm{mV}$ amplitude. The measurements were done in two-contacts mode, taking the two outer electrodes used for the DC resistance measurements with the sample in the same chip-carrier. The necessary calibration of the equipment to avoid spurious cables and sample holder contribution was done prior to the measurements and without the sample. Table I describes the studied samples, their length, resistance at $300 \mathrm{~K}$, and the annealing treatment.

First, we discuss the results obtained using the conventional four point measurements in order to avoid possible contributions from the contact barriers between the

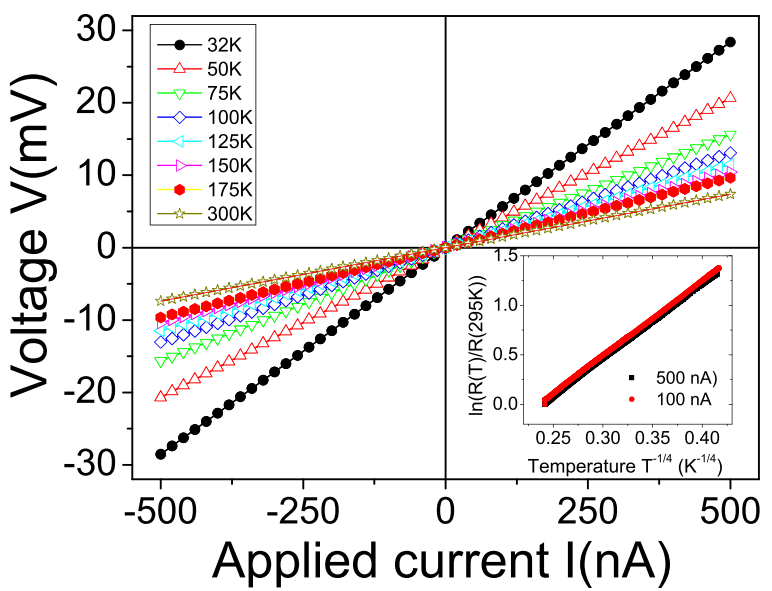

FIG. 2. Current-voltage $(I-V)$ curves at different temperatures for sample TiO2a measured with four contacts. Insert: Logarithm of the normalized resistance vs. $T^{-1 / 4}$ for sample TiO2a at two currents, $100 \mathrm{nA}$ and $500 \mathrm{nA}$. The observed linearity in the used scale indicates a 3D variable range hopping mechanism.

electrodes and the oxide surface. In this contact configuration, we observe that the $I-V$ curves are linear over all the temperature range, see Fig. 2. The $T$-dependence of the resistance $R(T)$, shown in the inset of Fig. 2, indicates that $R(T)=R_{0} \exp \left[\left(\frac{T_{0}}{T}\right)^{0.25}\right]$, with $R_{0}$ an arbitrary prefactor and $T_{0}$ a characteristic temperature coefficient. This dependence is compatible with the three dimensional (3D) variable range hopping (VRH) conduction mechanism known as the Mott law, a model that describes conduction in strongly disordered systems with localized charge-carrier states. ${ }^{22}$ According to this model, $T_{0}=18 / k_{B} \xi^{3} N\left(\mathrm{E}_{\mathrm{F}}\right)$, where $\xi$ is the localization length, $N\left(E_{F}\right)$ is the density of states at the Fermi level $E_{F}$, and $k_{B}$ the Boltzmann constant. From the observed dependence, see inset in Fig. 2, we obtain $T_{0} \simeq 3250 \mathrm{~K}$. Assuming that the localization length is of the order of $\approx 1 \mathrm{~nm},{ }^{23}$ we estimate $N\left(E_{F}\right) \simeq 6.4 \times 10^{28} \mathrm{ev}^{-1} \mathrm{~m}^{-3}$, a value comparable with those from literature. ${ }^{24}$

Figure 3 shows the results of the impedance spectroscopy measurements of sample $\mathrm{TiO} 2 \mathrm{a}$ at room temperature. Similar results were obtained for other samples. In general, for a semiconductor, different resistances and capacitances $(R, C)$ due mainly to grain boundaries $\left(R_{g b}, C_{g b}\right)$ and grain interior $\left(R_{g}, C_{g}\right)$ are assumed. Each conduction process is modeled by a parallel $R / C$ equivalent circuit. Such contributions can be in parallel or in series. ${ }^{25}$ The process-related contributions can be recognized through a cole-cole plot by two different arcs related to the grain boundary, at low frequencies, and grain interior at higher frequencies. In the present case, see Fig. 3(a), a single arc is observed, which can be fitted with the following equation:

TABLE I. Specifications of the investigated nanotubes. The shown lengths were measured between the voltage electrodes using scanning electron microscopy. Resistivity values range between $10^{-3}$ and $1 \Omega \mathrm{cm}$.

\begin{tabular}{|c|c|c|c|c|c|c|}
\hline & TiOla & TiOlb & $\mathrm{TiO} 2 \mathrm{a}$ & $\mathrm{TiO} 2 \mathrm{~b}$ & $\mathrm{TiO} 2 \mathrm{c}$ & $\mathrm{TiO} 3 \mathrm{~b}$ \\
\hline Length $(\mu \mathrm{m})$ & $1.5 \pm 0.1$ & $1.4 \pm 0.1$ & $0.8 \pm 0.1$ & $0.5 \pm 0.1$ & $1.5 \pm 0.1$ & $0.8 \pm 0.1$ \\
\hline Annealed(1 h,air) & $350^{\circ} \mathrm{C}$ & $350^{\circ} \mathrm{C}$ & $450^{\circ} \mathrm{C}$ & $450^{\circ} \mathrm{C}$ & $450^{\circ} \mathrm{C}$ & $450^{\circ} \mathrm{C}$ \\
\hline$R_{300 \mathrm{~K}}(\mathrm{k} \Omega)$ & $101.3 \pm 0.1$ & $27330 \pm 200$ & $14.7 \pm 0.1$ & $11.1 \pm 0.1$ & $116.7 \pm 0.1$ & $160 \pm 1$ \\
\hline
\end{tabular}



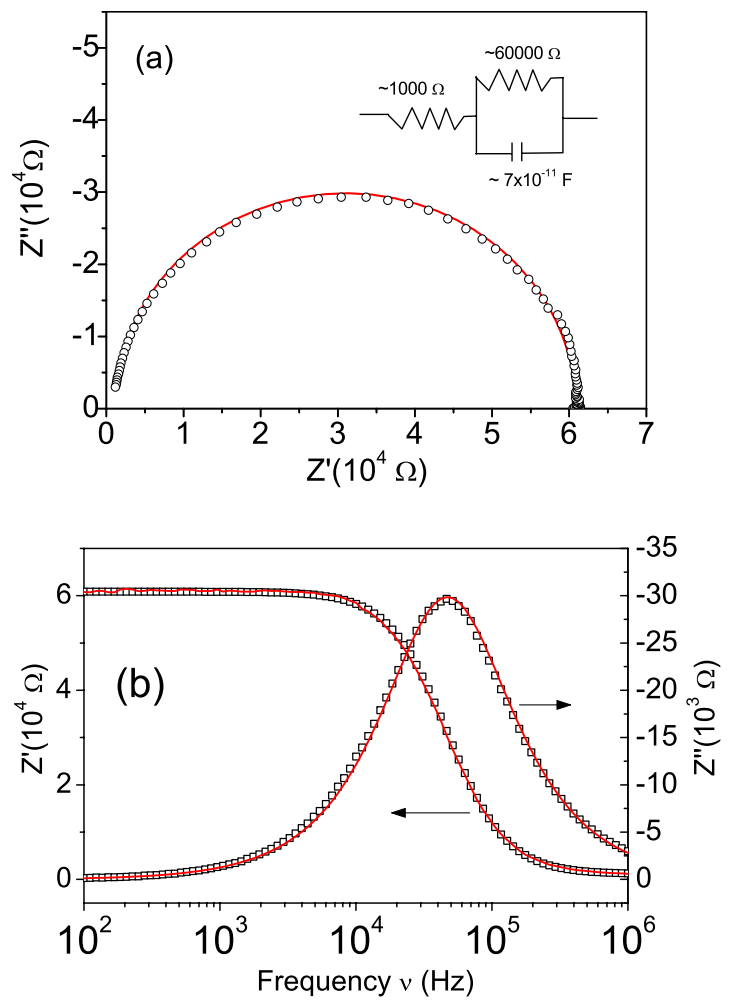

FIG. 3. (a) Nyquist plot of a single $\mathrm{TiO}_{2}$ of sample TiO2a. The red line is the fitting to the experimental data following Eq. (1) assuming the equivalent circuit shown in the inset. (b) Frequency dependence of $Z^{\prime}$ and $Z^{\prime \prime}$ with their corresponding fitting curves.

$$
Z^{\star}=Z^{\prime}+i Z^{\prime \prime}=R_{0}+\left(\frac{1}{R_{g, g b}}+i \omega C_{g, g b}\right)^{-1},
$$

where $R_{0}$ is the total resistance due to external component as wiring, $R_{g, g b}$ is the grain or grain boundary resistance, and $C_{g, g b}$ is the grain or grain boundary capacitance. From the fit of the data to Eq. (1), we obtained $R_{0}=\left(10^{3} \pm 50\right) \Omega, R_{g}$ $=(6 \pm 02) \times 10^{4} \Omega, C_{g}=(7 \pm 1) \times 10^{-11} \mathrm{~F}$. The value of the capacitance indicates a conduction process through the grain cores, ${ }^{26,27}$ in agreement with recently reported measurements in pellets of $\mathrm{TiO}_{2}$ nanotubes. ${ }^{28}$

Furthermore and for completeness, Fig. 3(b) shows the frequency dependence of $Z^{\prime}$ and $Z^{\prime \prime}$ of the studied sample. The observed behavior can be well fitted with the same equivalent circuit, as expected. It can be seen that there is a single mechanism of conduction since it is possible to see a single change of slope and a single minimum for the frequency dependence of $Z^{\prime}$ and $Z^{\prime \prime}$.

Note that the resistances measured in all the samples indicate resistivity values below $\sim 1 \Omega \mathrm{cm}$ at room temperature. These values are much smaller than the intrinsic resistivity of $\mathrm{TiO}_{2}$, very probably due to the large density of intragrain defects such as oxygen vacancies, known to influence the electrical properties of this oxide. ${ }^{28}$ These defects can facilitate the conduction within the grain, in spite of the existence of grain boundaries that do not have an appreciable contribution in the conduction process in our polycrystalline samples at room temperature.

As mentioned in the experimental section, the contacts were done by sputtering of chromium and gold. When chromium is sputtered on $\mathrm{TiO}_{2}$, an oxide is formed ${ }^{29}$ on the $\mathrm{TiO}_{2}$ surface such that an extra potential barrier is formed. Each of the nanotubes has been measured using the two-point method. An example can be seen for the TiO3b sample in Fig. 4. We get a clear linear ohmic behavior at room temperature whereas at temperatures below $\sim 150 \mathrm{~K}$ the contribution of an energy barrier is obvious and it becomes larger decreasing temperature. All other samples show similar $I-V$ curves, a fact that speaks for the general formation of a potential barrier and the inadequacy of the two points method to obtain the intrinsic transport properties of several oxides. The observed behavior in the $I-V$ curves can be understood taking into account the existence of a potential barrier and thermally activated process and the contribution of a temperature dependent ohmic term, given by the equation

$$
V=a(T) \tanh \left(I / I_{0}\right)+b(T) I,
$$

where $a(T), b(T)$, and $I_{0}$ are fitting parameters. In Fig. 4, we show the fits of the $I-V$ curves following Eq. (2). From these fits, we obtain that $a(T) \simeq 26[\mathrm{VK}] / \mathrm{T}, I_{0} \simeq 1 \mu \mathrm{A}$, and $b(30 \mathrm{~K}) \simeq 4.6 \times 10^{5} \Omega$, decreasing slightly with $T$.

The results of the temperature dependence of the resistance at different constant currents are shown in the inset of Fig. 4. At low input currents, the normalized resistance follows roughly an inverse temperature dependence due to the influence of the potential barrier, which is the reason for the non-linear $I-V$ characteristics shown in Fig. 4. Obviously, this influence decreases with temperature and current; it remains negligible at $T>150 \mathrm{~K}$, temperature range where the resistance shows an ohmic behavior.

Concluding, we have isolated $\mathrm{TiO}_{2}$ nanotubes within the micro- and nanometer scale and we were able to contact them using a combination between electron beam induced deposition and electron beam lithography for electronic transport measurements. The temperature dependent of the resistance is described by the Mott variable range hopping mechanism. The usage of $\mathrm{Cr} / \mathrm{Au}$ contacts for transport measurement at temperatures below $150 \mathrm{~K}$ does produce a measurable potential barrier. Impedance spectroscopy results

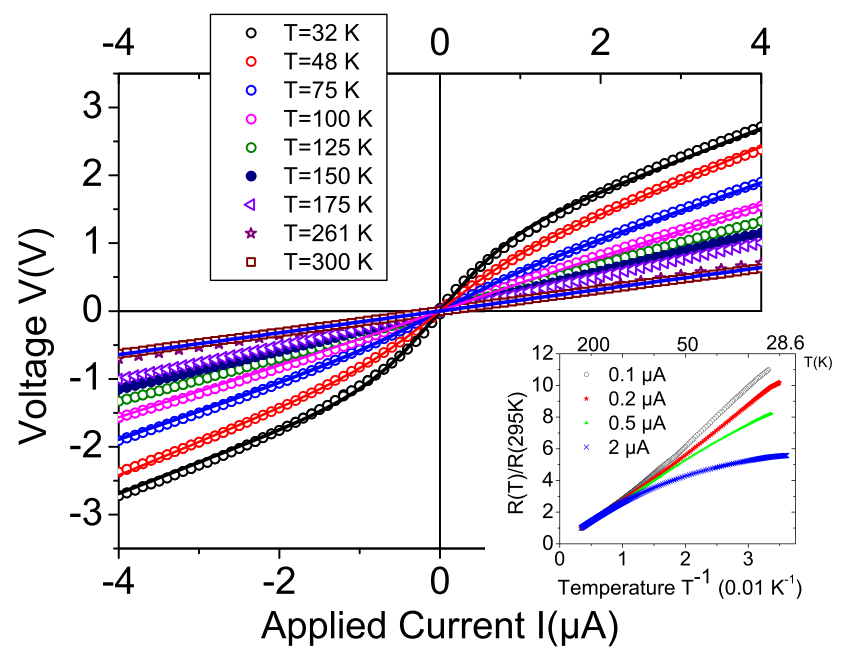

FIG. 4. Current-voltage characteristics for sample TiO3b. The continuous lines through the points at five different temperatures $(32 \mathrm{~K}, 48 \mathrm{~K}, 75 \mathrm{~K}$, $100 \mathrm{~K}$, and $300 \mathrm{~K}$ ) follow Eq. (2). Inset: Normalized resistance vs. inverse temperature for sample $\mathrm{TiO} 3 \mathrm{~b}$ at different constant currents. 
indicate that a single transport through the grain cores is active.

This work is being supported by the collaborative project SFB 762 "Functionality of Oxide Interfaces" and the DFG cluster of excellence "Engineering of Advanced Materials."

${ }^{1}$ K. I. Hadjiivanov and D. G. Klissurski, Chem. Soc. Rev. 25, 61 (1996).

${ }^{2}$ A. Fujishima and K. Honda, Nature 238, 37 (1972).

${ }^{3}$ B. O'Regan and M. Grätzel, Nature 353, 737 (1991).

${ }^{4}$ M.-H. Seo, M. Yuasa, T. Kida, J.-S. Huh, K. Shimanoe, and N. Yamazoe, Sens. Actuators B 137, 513 (2009).

${ }^{5}$ A. S. Aricò, P. Bruce, B. Scrosati, J. Tarascon, and W. van Schalkwijk, Nat. Mater. 4, 366 (2005).

${ }^{6}$ U. Diebold, Surf. Sci. Rep. 48, 53 (2003).

${ }^{7}$ X. Chen and S. S. Mao, Chem. Rev. 107, 2891 (2007).

${ }^{8}$ H. Tang, K. Prasad, R. Sanjinbs, P. E. Schmid, and F. Levy, J. Appl. Phys. 75, 2042 (1994).

${ }^{9}$ L. Forro, O. Chauvet, D. Emin, L. Zuppiroli, H. Berger, and F. Levy, J. Appl. Phys. 75, 633 (1994).

${ }^{10}$ J. M. Macak, M. Zlamal, J. Krysa, and P. Schmuki, Small 3, 300 (2007).

${ }^{11}$ X. Liu, P. K. Chu, and C. Ding, Mater. Sci. Eng. R 47, 49 (2004).

${ }^{12}$ P. Roy, S. Berger, and P. Schmuki, Angew. Chem. Int. Ed. 50, 2904 (2011).

${ }^{13}$ A. G. Muñoz, Electrochem. Acta 52, 4167 (2007).

${ }^{14}$ S. P. Albu, H. Tsuchiya, S. Fujimoto, and P. Schmuki, EJIC 2010, 4351 (2010).
${ }^{15}$ A. W. Czanderna, C. N. R. Rao, and J. M. Honig, Trans. Faraday Soc. 54, 1069 (1958).

${ }^{16}$ H. Mirabolghasemi, N. Liu, K. Lee, and P. Schmuki, Chem. Comm. 49, 2067 (2013).

${ }^{17}$ S. P. Albu, A. Ghicov, S. Aldabergenova, P. Drechsel, D. LeClere, G. E. Thompson, J. M. Macak, and P. Schmuki, Adv. Mater. 20, 4135 (2008).

${ }^{18}$ J. R. Macdonald and J. A. Garber, J. Electrochem. Soc. 124, 1022 (1977).

${ }^{19}$ M. Hattori, K. Noda, T. Nishi, K. Kobayashi, H. Yamada, and K. Matsushige, Appl. Phys. Lett. 102, 043105 (2013).

${ }^{20}$ M. Huth, F. Porrati, C. Schwalb, M. Winhold, R. Sachser, M. Dukic, J. Adams, and G. Fantner, Beilstein J. Nanotechnol. 3, 597 (2012).

${ }^{21}$ D. Spoddig, K. Schindler, P. Rödiger, J. Barzola-Quiquia, K. Fritsch, H. Mulders, and P. Esquinazi, Nanotechnology 18, 495202 (2007).

${ }^{22}$ N. F. Mott, J. Non-Cryst. Solids 1, 1 (1968).

${ }^{23}$ L. X. Chen, T. Rajh, Z. Wang, and M. C. Thurnauer, J. Phys. Chem. B 101, 10688 (1997).

${ }^{24}$ A. Yildiz, S. B. Lisesivdin, M. Kasap, and D. Mardare, Optoelectron. Adv. Mater.-Rapid Commun. 1, 531 (2007).

${ }^{25}$ S. M. Haile, D. L. West, and J. Campbell, J. Mater. Res. 13, 1576 (1998).

${ }^{26}$ Impedance Spectroscopy: Theory, Experiment, and Applications, edited by E. Barsoukov and J. R. MacDonald (John Wiley \& Sons Inc., New York, 2005).

${ }^{27}$ E. Chinarro, J. R. Jurado, F. M. Figueiredo, and J. Frade, Solid State Ionics 160, 161 (2003).

${ }^{28}$ P. P. Vijayan, M. Thomas, and K. C. George, J. Appl. Phys. 112, 104308 (2012).

${ }^{29} \mathrm{C}$. Winde, Herstellung und Charakterisierung von $\mathrm{Cr}$-Schichten auf $\mathrm{TiO}_{2}-(110)$ Oberflächen (Max-Planck-Institut für Metallforschung, Stuttgart, 2002). 